\section{Ventricular fibrillation after bortezomib therapy in a patient with systemic amyloidosis}

\author{
Satoshi Yamasaki, ${ }^{1,2}$ Tsuyoshi Muta, ${ }^{1}$ \\ Taiki Higo, ${ }^{3}$ Hirotake Kusumoto, ${ }^{1}$ \\ Eiko Zaitsu, ${ }^{3}$ Toshihiro Miyamoto, ${ }^{1}$ \\ Yoshinao Oda, ${ }^{4}$ Koichi Akashi ${ }^{1}$
}

${ }^{1}$ Department of Medicine and Biosystemic Sciences, School of Medical Sciences, Kyushu University, Fukuoka; 2Department of Hematology, National Hospital Organization Kyushu Medical Center, Fukuoka; ${ }^{3}$ Department of Cardiovascular Medicine, Faculty of Medicine, Kyushu University, Fukuoka; ${ }^{4}$ Department of Anatomic Pathology, Faculty of Medicine, Kyushu University, Fukuoka, Japan

\section{Abstract}

A 64-year-old female was diagnosed with systemic amyloidosis associated with multiple myeloma. Bortezomib and dexamethasonetherapy was initiated; however, she developed lethal ventricular fibrillation (VF) and cardiac arrest after 84 hours of therapy. Cardiopulmonary resuscitation using direct current shocks with epinephrine and amiodarone was initiated but failed to receive cardiac function. Although her arterial pulsations recovered immediately after the injection of vasopressin, she died of heart failure 8 hours after the onset of VF. Cardiac amyloidosis was verified by autopsy. Although the direct association of bortezomib with lethal VF remained to be clarified in our patient, the current report emphasizes on bortezomib as a substantial risk factor for cardiomyocyte damage. The potential risk of lethal events associated with cardiac amyloidosis should be carefully considered during bortezomib treatment for patients with $\mathrm{AL}$ amyloidosis.

\section{Introduction}

Systemic immunoglobulin light-chain (AL) amyloidosis is a disorder of plasma cells characterized by the deposition of amyloid fibrils in various organs, eventually leading to organ dysfunction and death. ${ }^{1}$ Cardiac involvement is observed in approximately $60 \%$ patients with AL amyloidosis. ${ }^{2}$ Once a diagnosis of cardiac amyloidosis has been established, survival time is limited. Of late, novel agents such as bortezomib have apparently become estab- lished as important drugs for the treatment of systemic AL amyloidosis. ${ }^{3}$

Here we present a case of systemic AL amyloidosis with severe cardiac involvement in a 64-year-old woman. We expected bortezomib to be efficacious but could not avoid the complication of fatal ventricular fibrillation (VF). In this case report, we evaluated the possibility of cardiac toxicity induced by bortezomib by investigating the clinical course of the patient and reviewing the relevant literature.

\section{Case Report}

A 64-year-old female suffering from general fatigue, appetite loss, and weight loss was referred to our institute for the investigation of M-protein and hypercalcemia. Upon hospital admission, her Eastern Cooperative Oncology Group performance status was 2 and her Karnofsky performance status (KPS) was approximately $50 \%$. She was also found to suffer from class II heart failure according to the functional classification system of New York Heart Association (NYHA). Physical examination revealed a palpable liver edge $15 \mathrm{~cm}$ below the costal margin and bilateral pretibial pitting edema.

Results of her blood test revealed the following: aspartate aminotransferase, 81 (normal, 13-33) U/L; alkaline phosphatase, 534 (115359) U/L; $\gamma$-glutamyl transpeptidase, 311 (1047) U/L; lactate dehydrogenase, 343 (119-229) $\mathrm{U} / \mathrm{L}$; calcium, 11.7 (8.7-10.3) mg/dL; creatinine, $1.18(0.4-0.7) \mathrm{mg} / \mathrm{dL}$; brain natriuretic peptide (BNP), 1630.5 (0-8.4) pg/mL; N-terminal prohormone of BNP (NT-proBNP), 8290 (0-125) $\mathrm{pg} / \mathrm{mL}$; and troponin T, $0.242(0-0.014) \mathrm{ng} / \mathrm{mL}$. Her serum beta- 2 microglobulin level was 5.5 $(0-2.5) \mathrm{mg} / \mathrm{L}$ and free light chain difference (FLC-diff) was $33.46 \mathrm{mg} / \mathrm{dL}$.

Immunoelectrophoresis demonstrated a monoclonal immunoglobulin light-chain with $\lambda$ isotype in the urine. A bone marrow aspirate revealed $10 \%$ plasma cells with $\mathrm{CD}^{+} 8^{+}$, $\mathrm{CD} 138^{+}$, and cytoplasmic $\lambda+$ phenotypes. An iliac biopsy confirmed the infiltration of plasma cells. Diffuse osteoporosis was revealed by radiography, and magnetic resonance imaging showed the absence of focal lesions. Histopathological examination revealed amyloid infiltration in biopsy samples of the gastric mucosa.

Marked cardiomegaly was noted on chest radiography. Electrocardiography (ECG) revealed left-axis deviation, low-voltage QRS complexes, and first degree atrioventricular block (Figure 1A). A 24-h Holter ECG demonstrated 129 beats of ventricular premature contractions (VPCs) and 1268 beats of atrial premature contractions (APCs), including 5 VPC short runs (maximum, 5 runs) and APC short
Correspondence: Satoshi Yamasaki, Department of Hematology, National Hospital Organization Kyushu Medical Center, 1-8-1 Jigyohama, Chuoku, Fukuoka 810-8563, Japan.

Tel. +81.928.520.700 - Fax: +81.928.478.802.

E-mail: yamas009@kyumed.jp

Key words: systemic amyloidosis, multiple myeloma, ventricular fibrillation, bortezomib.

Acknowledgments: the authors thank all participating physicians, nurses, and medical staff for their cooperation in the care and treatment of this patient.

Funding: this work was supported in part by a grant-in-aid from the Ministry of Education, Culture, Sports, Science and Technology of Japan (to K.A.) and a grant-in-aid from the Ministry of Health, Labor and Welfare of Japan (to K.A.).

Contributions: the authors contributed equally.

Conflict of interests: the authors declare no potential conflict of interests.

Received for publication: 23 May 2013.

Revision received: 26 June 2013.

Accepted for publication: 25 July 2013.

This work is licensed under a Creative Commons Attribution NonCommercial 3.0 License (CC BYNC 3.0).

(C) Copyright S. Yamasaki et al., 2013

Licensee PAGEPress, Italy

Hematology Reports 2013; 5:e12

doi:10.4081/hr.2013.e12

runs (maximum, 8 runs; Figure 1B). Transthoracic echocardiography revealed a left ventricular ejection fraction of $64 \%$, diffuse thickening of the left and right ventricular walls with granular sparkling, and pericardial effusion.

She was diagnosed with systemic AL amyloidosis associated with Bence Jones protein type multiple myeloma (MM). After written informed consent was obtained from the patient, administration of dexamethasone and bortezomib (oral dexamethasone, $20 \mathrm{mg} /$ day, days $1-4$; bortezomib, $1.3 \mathrm{mg} / \mathrm{m}^{2} /$ day, subcutaneous injection on day 4) was initiated under 24-h ECG monitoring (Figure 1C). The incidence of tachycardia was decreased through dexamethasone treatment. Serum calcium levels were corrected by intravenous infusions of normal $(0.9 \%)$ saline with elcatonin, leading to increased body weight. After adjustment of infusions and diuretic treatment, her body weight returned to that at baseline. The day before the onset of VF, her serum mineral levels were within normal limits as follows: calcium, $9.2 \mathrm{mg} / \mathrm{dL}$; magnesium, 1.8 (1.8-2.7) $\mathrm{mg} / \mathrm{dL}$; and potassium, $3.9(3.6-4.9) \mathrm{mmol} / \mathrm{L}$. The patient no longer complained of insomnia, 
constipation, or the stress of hospital admission. However, approximately $84 \mathrm{~h}$ after bortezomib administration, in the middle of the night, the patient conversed with nursing staff and walked to the rest room for urination. A few minutes later, her cardiac monitor alarm sounded and showed VF pattern. She was found lying down at her bedside. Cardiopulmonary resuscitation (CPR) using direct current shocks with epinephrine and amiodarone was initiated. Her serum mineral levels were as follows: sodium, 138.1 (138-146) mmol/L; potassium, $3.56 \mathrm{mmo} / \mathrm{L}$; ionized calcium, 1.11 (1.11.4) $\mathrm{mmol} / \mathrm{L}$; and bicarbonate, 29.9 (22-30)
mmol/L. Continuous CPR for approximately 30 min failed to recover the cardiac features required to sustain the general circulation, but just after the injection of vasopressin, her arterial pulsations recovered. However, she died of heart failure $8 \mathrm{~h}$ after the onset of VF.

Autopsy revealed congested, hemorrhagic lungs and right and left ventricular hypertrophy (heart weight, $\geq 400 \mathrm{~g}$ ). Gross examination revealed round-to-web-like yellow waxy deposits in the myocardium. Histopathological examination revealed cardiac amyloid deposition, even in the atrioventricular node, and no ischemic lesion was complicated (Figure 1D,
E). The amyloidosis had also spread to the tongue, lung, liver, colon, kidney, bone marrow, adrenal gland, thyroid gland, and ovaries.

\section{Discussion}

The present patient possessed one of two risk factors (systolic blood pressure $<100$ $\mathrm{mmHg}$ ) in stage III cardiac amyloidosis. ${ }^{4}$ According to the revised prognostic staging system for AL amyloidosis, the present patient is categorized to stage 3 (NT-ProBNP $\geq 1800$
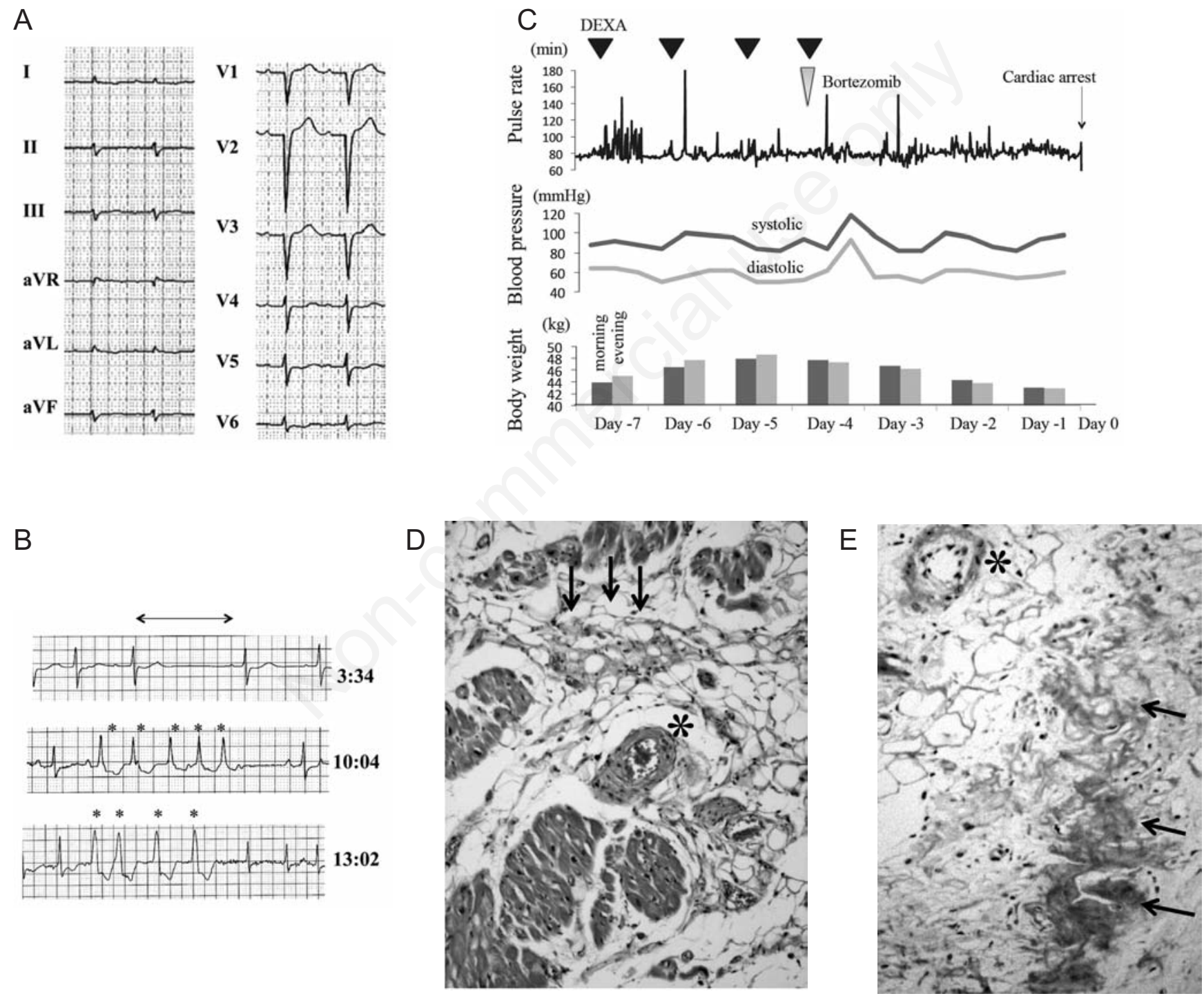

Figure 1. A) Low-voltage of QRS complexes and the absence of $P$ wave in all leads on electrocardiography (ECG) performed on hospital admission. B) The results of 24-h Holter ECG reveal a long pause (arrow) and short-run of premature contractions (asterisks). C) Results from a continuous bed-side monitoring device during 1 week around bortezomib subcutaneous injections. The day when the cardiac arrest occurred is referred to as day 0. DEXA, dexamethasone. D) Histopathological analyses of the region of atrioventricular nodes. Deposits of amorphous materials (arrows) can be seen in a hematoxylin and eosin stained section (x100). E) The amorphous deposits (arrows) are stained positively by Congo-red (x100). The asterisks indicate perivascular amorphous materials that are positively stained with Congo-red. 
Table 1. Patients with heart failure after bortezomib treatment for multiple myeloma.

\begin{tabular}{|c|c|c|c|c|c|c|c|c|}
\hline Case & Age/sex & $\begin{array}{c}\text { Dose of Bor } \\
\left(\mathrm{mg} / \mathrm{m}^{2}\right)\end{array}$ & $\begin{array}{l}\text { Concurrent } \\
\text { therapy }\end{array}$ & $\begin{array}{l}\text { No. of Bor } \\
\text { therapies }\end{array}$ & $\begin{array}{l}\text { Cumulative dose } \\
\text { of Bor }\left(\mathrm{mg} / \mathrm{m}^{2}\right)\end{array}$ & $\begin{array}{c}\text { Onset } \\
\text { (days after } \\
\text { Bor treatment) } \\
\text { of heart failure }\end{array}$ & Outcome & Reference \\
\hline 1 & $79 / \mathrm{F}$ & 0.7 & Dex & 1 & 0.7 & 5 & Alive & 8 \\
\hline 2 & $70 / \mathrm{F}$ & 1.3 & Dex & 22 & 28.6 & 3 & Alive & 9 \\
\hline 3 & $56 / F$ & 1.3 & Dex & 16 & 20.8 & ND & Alive & 10 \\
\hline 4 & 73/M & 1.3 & ND & 24 & 31.2 & ND & Alive & 11 \\
\hline 5 & $61 / F$ & 1.3 & ND & 16 & 20.8 & ND & Alive & 11 \\
\hline 6 & $71 / \mathrm{M}$ & 1.3 & ND & 16 & 20.8 & ND & Alive & 11 \\
\hline 7 & $80 / \mathrm{F}$ & 1.3 & ND & 16 & 20.8 & ND & Alive & 11 \\
\hline 8 & $64 / \mathrm{F}$ & 1.3 & Dex & 1 & 1.3 & 3 & Dead & This report \\
\hline
\end{tabular}

Bor, bortezomib; Dex, Dexamethasone; ND, no data.

$\mathrm{pg} / \mathrm{mL}$, and FLC-diff $\geq 18 \mathrm{mg} / \mathrm{dL}){ }^{5}$ Both studies indicated that the survival curves of these most advanced stage III patients had dropped steeply at first 6 months after diagnosis., Therefore, the risk of developing lethal arrhythmia due to cardiac amyloidosis is estimated to be extremely high in the present patient. In addition, if we exclude the other possible causes of KPS values, vital signs, blood examination results, and documented infections, the direct trigger of VF seemed to be ascribed to vasovagal syncope after urination. It is true that the largest study conducted with bortezomib in multiple myeloma showed minimum adverse effect of bortezomib on the incidence of heart failure. ${ }^{6}$ However, concerning about AL myloidosis, the adverse cardiac events at grade $\geq 3$ occurred more frequently in the $1.3 \mathrm{mg} / \mathrm{m}^{2}$ twice-weekly group (29\%) than the $1.6 \mathrm{mg} / \mathrm{m}^{2}$ once-weekly group (6\%). ${ }^{7}$ Accordingly, we could not overlook the timecourse between the injection of bortezomib and the occurrence of $\mathrm{VF}$ in the present patient. In the literatures, seven patients suffered congestive heart failure 3-5 days after bortezomib administration and recovered within 2 weeks (Table 1). ${ }^{8-11}$ Like our patient, a patient has been complicated with cardiac dysfunction after the first administration of bortezomib. ${ }^{8}$ A rat model revealed that reversible cardiac damages occurred within 1 week of bortezomib treatment. ${ }^{12}$ Taken together, some adverse effects involving cardiomyocytes may occur at some interval after bortezomib administration.

The efficacy of bortezomib for AL amyloidosis has been also proposed. ${ }^{7,13}$ However, there are some notable events that took place during these trials. One of the 67 patients in the former trial was complicated with acute heart failure during cycle 2 of botezomib, resulting in death 2 days after the last dose of the drug. ${ }^{7}$ This patient had a history of coronary artery disease and heart failure. ${ }^{7}$ In the latter trial, after 3 cycles of cyclophosphamide, bortezomib, and dexamethasone, 1 patient was complicated with fluid overload while another patient developed decompensated heart failure. ${ }^{13}$ Although the authors did not mention the possibility of accidental cardiac event independent of bortezomib nor the association between bortezomib and heart failure, we cannot deny the cardiac arrest induced by bortezomib.

According to the data in vitro, the ubiquitinproteasome system (UPS) is activated in hypertrophic cardiomyocytes, ${ }^{14}$ and it protects blood vessels from the formation and rupture of plaques, ${ }^{9}$ and maintains protein quality in cardiaomyocytes. ${ }^{15}$ With regard to dilated cardiomyopathy, the genes for UPS are highly expressed in a rat model and human heart specimens. ${ }^{16}$ Age-related activation of the UPS has been proposed to maintain essential cardiac stroke. ${ }^{17}$ Once proteasome activity is inhibited, atherosclerotic plaques become unstable, smooth muscle cells become apoptotic, and expression of the adrenergic sensitizer GRK2 increases, which can harm cardiaomyocytes. The inhibition of nuclear factor-kappa $\mathrm{B}$ by bortezomib is responsible for higher incidence of cardiac damage in AL amyloidosis compared to multiple myeloma. ${ }^{18}$ In the future, we should determine the mechanism that could have been associated with lethal VF in our patient.

We should discuss about the use of implantable devices and CPR. Our patient did not have a history of syncope, and then no indication for preventive implantation of a pacemaker. According to a trial, 19 patients associated with syncope and/or $\geq 2$ consecutive VPC beats received an implantable cardioverterdefibrillator (ICD), and 6 of them died from electromechanical dissociation. ${ }^{19}$ In our patient, immediate direct-current shocks were not effective for recovery from cardiac arrest. Accordingly, whether we could have avoided the occurrence of lethal VF in our patient by
ICD implantation remains unclear. With regard to CPR, vasopressin has been considered to be a promising alternative to epinephrine and proposed as an initial vasopressor for cardiac arrest. ${ }^{20}$ Vasopressin is associated with a longer duration of action compared with epinephrin. Our patient required frequent injections of epinephrine, one shot of vasopressin could have maintained her blood pressure for a long period, and then this may have been effective in maintaining the systemic circulation.

\section{Conclusions}

We described a form of systemic AL amyloidosis with cardiac involvement that resulted in sudden cardiac death induced by VF following bortezomib therapy. We should not ignore the potential risk of lethal arrythmia associated with advanced cardiac amyloidosis during bortezomib treatment. Futhermore, vasopressin may be useful for CPR in patients with cardiac amyloidosis requiring frequent injection of epinephrine.

\section{References}

1. Merlini G, Bellotti V. Molecular mechanisms of amyloidosis. N Engl J Med 2003; 349:583-96.

2. Obici L, Perfetti V, Palladini G, et al. Clinical aspects of systemic amyloid diseases. Biochim Biophys Acta 2005;1753: 11-22.

3. Gatt ME, Palladini G. Light chain amyloidosis 2012: a new era. Br J Haematol 2013;160:582-98.

4. Wechalekar AD, Schonland SO, Kastritis E, et al. European collaborative study of treatment outcomes in 346 patients with car- 
diac stage III AL amyloidosis. Blood 2013;121:3420-7.

5. Kumar S, Dispenzieri A, Lacy MQ, et al. Revised prognostic staging system for light chain amyloidosis incorporating cardiac biomarkers and serum free light chain measurements. J Clin Oncol 2012; 30:989-95.

6. Richardson PG, Sonneveld P, Schuster MW, et al. Bortezomib or high-dose dexamethasone for relapsed multiple myeloma. $\mathrm{N}$ Engl J Med 2005;352:2487-98.

7. Reece DE, Hegenbart U, Sanchorawala V, et al. Efficacy and safety of once-weekly and twice-weekly bortezomib in patients with relapsed systemic AL amyloidosis: results of a phase 1/2 study. Blood 2011; 118:865-73.

8. Takamatsu H, Yamashita T, Kotani T, et al. Ischemic heart disease associated with bortezomib treatment combined with dexamethasone in a patient with multiple myeloma. Int J Hematol 2010;91:903-6.

9. Gupta A, Pandey A, Sethi S. Bortezomibinduced congestive cardiac failure in a patient with multiple myeloma. Cardiovasc
Toxicol 2012;12:184-7.

10. Bockorny M, Chakravarty S, Schulman P, et al. Severe heart failure after bortezomib treatment in a patient with multiple myeloma: a case report and review of the literature. Acta Haematol 2012;128:244-7.

11. Enrico 0, Gabriele B, Nadia C, et al. Unexpected cardiotoxicity in haematological bortezomib treated patients. $\mathrm{Br} \mathrm{J}$ Haematol 2007;138:396-7.

12. Nowis D, Maczewski M, Mackiewicz U, et al. Cardiotoxicity of the anticancer therapeutic agent bortezomib. Am J Pathol 2010;176:2658-68.

13. Venner CP, Lane T, Foard D, et al. Cyclophosphamide, bortezomib, and dexamethasone therapy in AL amyloidosis is associated with high clonal response rates and prolonged progression-free survival. Blood 2012;119:4387-90.

14. Voortman J, Giaccone G. Severe reversible cardiac failure after bortezomib treatment combined with chemotherapy in a nonsmall cell lung cancer patient: a case report. BMC Cancer 2006;6:129.

15. Hacihanefioglu A, Tarkun P, Gonullu E.
Acute severe cardiac failure in a myeloma patient due to proteasome inhibitor bortezomib. Int J Hematol 2008;88:219-22.

16. Weekes J, Morrison $\mathrm{K}$, Mullen A, et al. Hyperubiquitination of proteins in dilated cardiomyopathy. Proteomics 2003;3:20816.

17. Bulteau AL, Szweda LI, Friguet B. Agedependent declines in proteasome activity in the heart. Arch Biochem Biophys 2002;397:298-304.

18. Jancso G, Cserepes B, Gasz B, et al. Effect of acetylsalicylic acid on nuclear factorkappaB activation and on late preconditioning against infarction in the myocardium. J Cardiovasc Pharmacol 2005;46:295301.

19. Kristen AV, Dengler TJ, Hegenbart U, et al. Prophylactic implantation of cardioverterdefibrillator in patients with severe cardiac amyloidosis and high risk for sudden cardiac death. Heart Rhythm 2008;5:23540 .

20. Larabee TM, Liu KY, Campbell JA, et al. Vasopressors in cardiac arrest: a systematic review. Resuscitation 2012;83:932-9. 